\title{
Upper limits to absorption enhancement in thick solar cells using diffraction gratings
}

\author{
A. Mellor*, I. Tobías, A. Martí, M.J. Mendes and A. Luque \\ Instituto de Energía Solar, Universidad Politécnica de Madrid, Madrid 28040, Spain
}

\begin{abstract}
The application of diffraction gratings to solar cells is a promising approach to superseding the light trapping limits of conventional Lambertian structures. In this paper a mathematical formalism is derived for calculating the absorption that can be expected in a solar cell equipped with a diffraction grating, which can be applied to any lattice geometry and grating profile. Furthermore, the formalism is used to calculate the upper limit of total absorption that can theoretically be achieved using a diffraction grating. The derived formalism and limits are valid when the solar cell thickness is greater than the coherence length of the illuminating solar spectrum. Comparison is made to the upper limit achievable using an angularly selective Rugate filter, which is also calculated. Both limits are found to be considerably higher than the Lambertian limit within the range of sunlight concentration factors practically employed in photovoltaic systems $(1-1000 \times)$. The upper limit of absorption using the diffraction grating is shown to be equal to the thermodynamic limit for all absorbances and concentration factors. The limit for the Rugate filter is generally lower, but tends to the thermodynamic limit for lower cell absorbances. Copyright (C) 2011 John Wiley \& Sons, Ltd.
\end{abstract}

\section{KEYWORDS}

light trapping; diffraction gratings; absorption enhancement

\section{*Correspondence}

A. Mellor, Instituto de Energía Solar, Universidad Politécnica de Madrid, Madrid 28040, Spain. E-mail: mellor_alex@hotmail.com

\section{INTRODUCTION}

The purpose of this paper is to formulate mathematically the optical behaviour inside a solar cell with a diffraction grating attached to either face, and to apply the formalism to analytical calculation of the upper limit of absorption enhancement using a diffraction grating. The formalism and results are valid in the limit where the solar cell thickness is greater than the coherence length of the illuminating solar spectrum.

The use of optical designs to enhance absorption, known as light trapping, is of interest for solar cells that absorb a certain part of the solar spectrum weakly. Historically, this has been of major importance for thin film cells, and to a lesser extent, for bulk cells based on indirect semiconductors such as crystalline silicon. A more recent solar cell technology in need of light trapping is the quantum dot intermediate band solar cell (QD-IBSC): a particular implementation of the intermediate band solar cell (IBSC) concept [1]. The IBSC is capable of generating photocurrent from sub-bandgap photons due to the existence of a metallic intermediate band between the valence band and the conduction band. In a QD-IBSC, the intermediate band is formed from the confined states of quantum dots, the absorption in which is extremely weak. QD-IBSCs are fabricated by epitaxial growth on crystalline wafer substrates [2]. This presents renewed motivation for the study of light trapping in bulk structures.

Light trapping is conventionally realised by texturing of either or both of the cell surfaces. One approach is rough texturing [3], which tends to produce close to isotropic (Lambertian) scattering. It has been shown that if the surface is sufficiently textured, there is a tendency towards an absorption enhancement factor of $4 n^{2}$; the so called Lambertian limit [4]. For mono-crystalline Si solar cells, another approach is anisotropic etching of the $(1,0,0)$ oriented surface to reveal the close packed $(1,1,1)$ and $(1,1,-1)$ planes. This leads to a surface of square based pyramids, which are shown to scatter light into certain angles favourably [5]. In Ref. [6], a number of surface geometries based on this approach were studied theoretically using ray tracing. A tendency toward the Lambertian 
limit was found, the best studied structure exhibiting a 4\% higher short-circuit current enhancement than a Lambertian structure for the studied solar cell parameters.

The overall thermodynamic limit of light trapping has been found to be significantly higher than the Lambertian limit in all cases other than for a very weakly absorbing cell under isotropic illumination [7]. This has motivated the study of alternative light trapping schemes. One such scheme employs a diffraction grating on either cell surface to scatter incident light into laterally propagating diffraction orders with high optical path lengths. Thick solar cells equipped with gratings have been fabricated $[8,9]$, and their light trapping properties have been studied numerically [10-12].

In Section 2 of this paper we derive a mathematical formalism for calculating the absorption enhancement from the far-field grating efficiencies; this is appropriate in bulk structures where most absorption takes place in the far-field of the grating. A simple transfer matrix method is employed in which all re-interactions of confined orders with the grating are considered. We assume the cell to be illuminated by a cone of light; this corresponds to direct solar illumination or illumination from a concentrator. The method may be applied to a solar cell equipped with an arbitrary diffraction grating on the front or rear surface.

In Section 3, the formalism developed in Section 2 is applied to analytical calculation of the upper limit of light trapping using a diffraction grating, and determination of the conditions under which this limit can be met. For comparison, the upper limit of light trapping using a Rugate filter is also calculated. This alternative light trapping scheme combines a Lambertian scatterer with an angularly-selective photonic crystal (Rugate filter) on the front surface [13]. Analyses are made both in the limit of very weak absorption and for the more general case where absorption effects are more significant. In both instances, comparison is made to the Lambertian baseline and to the overall thermodynamic limit.

\section{MATHEMATICAL FORMULATION OF THE GRATING PROBLEM; CALCULATING THE ABSORPTION FROM THE SCATTERING MATRIX}

Figure 1a and b shows solar cell structures in which a diffraction grating is located on the front face (configuration (a)) and rear face (configuration (b)), respectively. In each case a perfect reflector is placed at the rear. In configuration (b), it is assumed there is zero reflectivity at the front surface. The coordinate axis is such that the cell faces and the grating lie in the $x y$ plane. The grating's lattice geometry may be either a uni-periodic line grating, or a bi-periodic square or triangular lattice. It may have any profile (by profile we mean the shape of the periodically repeated grating elements). It is assumed that all constituent materials of the grating are lossless (either

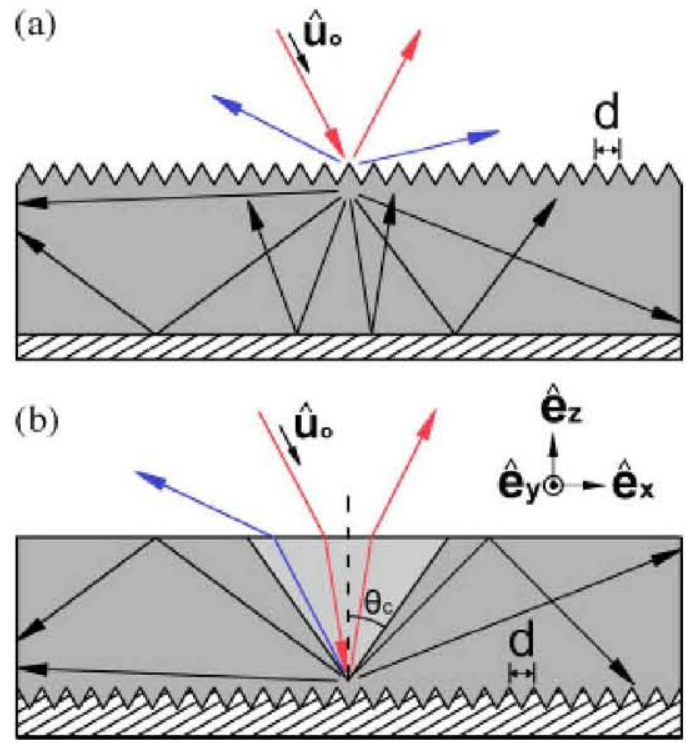

Figure 1. Two possible solar cell structures. In configuration (a) the grating is placed on the front face of the solar cell and in configuration (b) it is placed on the rear. A perfect reflector is placed at the rear in both. Confined orders, source-illuminated escaping orders and non-source-illuminated escaping orders are represented by black, red and blue arrows, respectively. In configuration (b) the lighter shaded triangular area represents the escape cone, which is defined by the critical angle of the front surface interface $\theta_{c}$.

perfect dielectrics or perfect conductors). Hence power dissipation in the grating and generation of surface plasmons is ignored.

\subsection{Plane-wave illumination}

In this subsection the solar cell is considered to be illuminated by a plane wave incident from above whose unit propagation direction vector in a vacuum is $\hat{\mathbf{n}}_{0}=u_{x 0} \mathbf{e}_{x}+u_{y 0} \mathbf{e}_{y}+u_{z 0} \mathbf{e}_{z}$, where $\mathbf{e}_{x}, \mathbf{e}_{\mathrm{y}}, \mathbf{e}_{z}$ are unit vectors parallel to the coordinate axes and $u_{x}, u_{y}, u_{z}$ are the so called direction cosines made with those axes. In either configuration the wave is incident on the grating and is diffracted into a number of reflected and transmitted propagating orders. The vector components of these orders tangential to the $x y$-plane $\left(\overrightarrow{\mathbf{u}}_{\|}=u_{x} \mathbf{e}_{x}+u_{y} \mathbf{e}_{y}\right)$ are given by the Fraunhofer equation:

$$
n \overrightarrow{\mathbf{u}}_{\|\left(m_{1}, m_{2}\right)}=\frac{m_{1} \overrightarrow{\mathbf{b}}_{1} \lambda_{0}}{2 \pi}+\frac{m_{2} \overrightarrow{\mathbf{b}}_{2} \lambda_{0}}{2 \pi}+\overrightarrow{\mathbf{u}}_{\| 0}
$$

$\overrightarrow{\mathbf{b}}_{1}$ and $\overrightarrow{\mathbf{b}}_{2}$ are the reciprocal lattice vectors of the diffraction grating $\left(\overrightarrow{\mathbf{b}}_{1}=\overrightarrow{0}\right.$ for a uni-periodic line grating) and $\left(m_{1}, m_{2}\right)$ are the integer diffraction order indices. $n$ is the refractive index of the medium in which the order propagates and $\lambda_{0}$ and $\overrightarrow{\mathbf{u}}_{\| 0}$ are the wavelength and tangential direction vectors in a vacuum. Equation (1) describes all propagating orders in either configuration. In configuration (a) there are 
reflected and transmitted orders whereas in configuration (b) there are only reflected orders. In configuration (a) all reflected orders escape and all transmitted orders, which propagate internal to the cell, are confined by the rear reflector. In configuration (b) all internal orders that make an angle with the $z$ axis that is less than the critical angle $\theta_{\mathrm{c}}$ escape; all which make an angle greater than $\theta_{\mathrm{c}}$ are confined at the front surface by total internal reflection. This defines the escape cone which is represented by the lighter shaded triangular area in (b). All confined orders are represented by black arrows.

In both configurations all confined rays re-impinge on the grating, their tangential component unchanged. These produce further sets of diffracted orders, the directions of which are identical to the original set due to the discrete translational symmetry of (1) [14]. This follows for all subsequent steps; radiant power is redistributed between orders at each diffraction event, but orders that are distinct from the original set are never created. The problem can therefore be formulated as a discrete system of $N$ diffracted orders which can be decomposed into $s$ and $p$ polarizations giving $2 N$ polarized diffracted orders. These can be divided into confined and escaping orders. For the purpose of the formalism all orders are considered to be diffracted from and incident on the grating. The initially incident plane wave is considered to be an incident order whose diffracted counterpart is the reflected zero order $\left(m_{1}=m_{2}=0\right.$ in Equation (1)) which has the same $\overrightarrow{\mathbf{u}} \|$ and therefore escapes the solar cell. This pair is denominated a sourceilluminated escaping order and is shown as two red arrows in Figure 1. All other escaping orders are denominated non-source-illuminated escaping orders and are incident on the grating with zero amplitude; these are represented by blue arrows in Figure 1.

Following conventional grating theory, the complex electric field amplitude of the $i$ th polarized order immediately after diffraction from the grating is linearly related to that of all other polarized orders immediately before incidence on the grating: the coefficients of this linear equation are the elements of the scattering matrix $\mathbf{S}=\left[S_{i, j}\right]_{2 N \times 2 N}$, which are complex and hence act on the amplitude and phase of the time-harmonic field [15].

$$
E_{i}^{\mathrm{diff}}=\sum_{1 \leq i \leq 2 N} S_{i, j} E_{j}^{\mathrm{inc}}
$$

The scattering matrix is a property of the specific grating profile and depends on the incident wavevector. There are a variety of methods available to calculate the scattering matrix computationally, based on rigorous solution of Maxwell's equations [16].

\subsection{Conical illumination}

We now consider the solar cell to be illuminated by a cone of light whose axis is aligned with the $z$ axis. This corresponds to direct illumination from the solar disc in a flat panel system with a solar tracker, or illumination from a two-axis solar concentrator. Diffuse illumination is ignored Conical illumination can be considered as illumination by a manifold of plane waves. The waves in the manifold are diffracted as described above producing distinct sets of diffracted orders which themselves constitute diffracted cones [14]. The tangential components $\left(u_{x}, u_{y}\right)$ of the cones diffracted from a triangular lattice grating, which are internal to the solar cell substrate, are depicted as filled circles in Figure 2, for different values of the wavelength to grating period ratio $\lambda / \Lambda$. On decreasing $\lambda / \Lambda$ the number of diffracted cones increases until there are sufficient cones to fill all directions within the cell completely (Figure 2d).

The scattering matrix takes a different form for each wave in the incident manifold. To treat conical illumination it is therefore necessary to divide the illumination cone into sub-manifolds across which there is very little variation in the scattering matrix elements. Each sub-manifold can then be considered as a discrete system. When the wavelength to grating period ratio $\lambda / \Lambda$ is small and diffracted orders are densely spaced, some orders of certain sub-manifolds may fall within the illumination cone and therefore coincide with another sub-manifold of the illumination cone. These must then be considered as pertaining to a single system which has multiple source-illuminated orders.

\subsection{Brightness calculations}

The brightness (defined as power flux per unit area per unit solid angle - also known as radiance) in a ray of

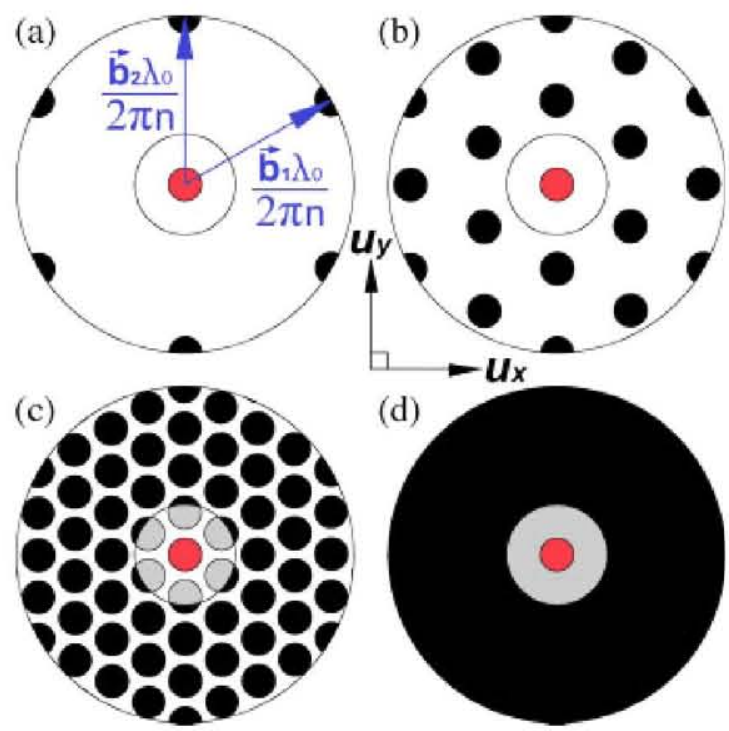

Figure 2. Direction cosine plots of illuminated directions internal to the cell for different wavelength to grating period ratios: (a) $\lambda /$ $n \Lambda=0.87$, (b) $\lambda / n \Lambda=0.43$, (c) $\lambda / n \Lambda=0.22$ and (d) $\lambda / n \Lambda<0.15$. The red, black and grey filled circles represent the illumination cone and the confined and escaping diffracted cones, respectively. The larger circle around the red filled circle is the escape cone which is only relevant when the grating is placed on the rear. 
infinitesimal solid angle $\mathrm{d} \Omega$ is related to the intensity $I$ and hence electric field amplitude $E$ by [17]:

$$
B=\frac{I}{\mathrm{~d} \Omega}=\frac{n \varepsilon_{0}^{2}|E|^{2}}{2 \mathrm{~d} \Omega}
$$

where $\varepsilon_{0}$ is the vacuum permittivity. The brightnesses of diffracted rays can therefore be calculated by substituting (2) into (3). The modulus squared of the sum in Equation (2) contains a number of interference terms between the fields of different incident orders. If the cell thickness is greater than the coherence length of the light inside the cell, the orders lose coherence between diffraction from and incidence on the grating. The statistical net contribution from all interference terms is then zero [17]. Hence, the brightnesses of incident and diffracted orders can be related by:

$$
\frac{B_{i}^{\text {diff }}}{n_{i}^{2}}=n_{i} u_{z i} \sum_{1 \leq j \leq 2 N} \frac{1}{n_{j} u_{z j}}\left|S_{i, j}\right|^{2} \frac{B_{j}^{\text {inc }}}{n_{j}^{2}}
$$

$n_{i}$ is the refractive index in which the $i$ th order propagates and $u_{z i}$ the direction cosine the $i$ th order makes with the $z$ axis. In matrix form this is

$$
\mathbf{B}^{\text {diff }}=\mathbf{U} \mathbf{H U}^{-1} \mathbf{B}^{\text {inc }}:=\mathbf{R} \mathbf{B}^{\text {inc }}
$$

$\mathbf{B}^{\text {diff }}$ and $\mathbf{B}^{\text {inc }}$ are $2 N$ dimensional vectors with elements $B_{i}^{\mathrm{diff}} / n_{i}^{2}$ and $B_{i}^{\mathrm{inc}} / n_{i}^{2}$, respectively. $\mathrm{U}$ is a diagonal matrix with $i$ th element $n_{i} \quad u_{z i}$ and $\mathbf{H}=\left[\left|S_{i, j}\right|^{2}\right]_{2 N \times 2 N}$. Equation (5) defines what we denominate the redistribution matrix $\mathbf{R}$.

The confined orders undergo a double pass of the solar cell active layer before returning to the grating. The active layer is modelled as a homogeneous material of width $w$ and absorption coefficient $\alpha$.The brightness of the $i$ th incident confined order is then given by the Lambert-Beer law:

$$
B_{i}^{\text {inc }}=B_{i}^{\text {diff }} \exp \left(-2 \alpha l_{i}\right) i \in \text { confined orders }
$$

where $l_{i}=w / u_{z i}$ is the length travelled by the $i$ th order in a single pass across the active layer.

All incident non-source-illuminated orders are fictitious and have zero brightness:

$$
B_{i}^{\text {inc }}=0 \quad i \in \begin{gathered}
\text { non-source-illuminated } \\
\text { escaping orders }
\end{gathered}
$$

Finally, the total escaping power leaving the grating is equal to the power incident on the grating from the source less the power absorbed in confined orders. As all diffracted orders have the same product of étendue and refractive index squared, this implies:

$$
\sum_{\substack{\text { source } \\ \text { illuminated } \\ \text { orders }}} \frac{B_{i}^{\text {inc }}}{n_{i}^{2}}=\sum_{\substack{\text { escaping } \\ \text { orders }}} \frac{B_{j}^{\text {diff }}}{n_{j}^{2}}+\sum_{\substack{\text { confined } \\ \text { orders }}} \frac{B_{j}^{\text {diff }}}{n_{j}^{2}}\left(1-\exp \left(-2 \alpha l_{j}\right)\right)
$$

In configuration (b) of Figure 1, source-illuminated orders make a single pass through the active layer before reaching the grating and suffer different attenuations depending on their trajectory. Taking this into account (8) can be reduced to:

$$
\begin{aligned}
& \frac{B_{i}^{\text {inc }}}{n_{i}^{2}}=\frac{\exp \left(-\alpha l_{i}\right)}{\sum_{\substack{\text { source } \\
\text { illuminated } \\
\text { orders }}} \exp \left(-\alpha l_{k}\right)} \\
& \left(\sum_{\substack{\text { escaping } \\
\text { orders } \\
\text { source }}} \frac{B_{j}^{\text {diff }}}{n_{j}^{2}}+\sum_{\begin{array}{c}
\text { confined } \\
\text { orders }
\end{array}} \frac{B_{j}^{\text {diff }}}{n_{j}^{2}}\left(1-\exp \left(-2 \alpha l_{j}\right)\right)\right) \\
& i \in \text { illuminated } \\
& \quad \text { orders }
\end{aligned}
$$

which is applicable to configuration (a) by considering that in this case the distance $l_{i}$ travelled through the active layer is zero for all source-illuminated orders.

Equations (6), (7) and (9) constitute a linear map from $\mathbf{B}^{\text {diff }}$ to $\mathbf{B}^{\text {inc }}$ :

$$
\mathbf{B}^{\text {inc }}=\mathbf{C B}^{\text {diff }}=\mathbf{C R B}^{\text {inc }}
$$

where $\mathbf{C}$ is the confinement matrix which can be partitioned into 6 blocks:

$$
\begin{aligned}
& \text { escaping confined } \\
& \text { orders orders } \\
& C=\left[\begin{array}{cc}
\underbrace{C_{1}}_{1} & \underbrace{C_{2}}_{2} \\
0 & 0 \\
0 & C_{3}
\end{array}\right]-\left\{\begin{array}{l}
\text { source illuminated } \\
\text { escaping orders } \\
\text { non source illuminated } \\
\text { escaping orders }
\end{array}\right.
\end{aligned}
$$

Sub-matrices $\mathbf{C}_{1}, \mathbf{C}_{2}$ and $\mathbf{C}_{3}$ are defined by:

$$
\begin{gathered}
C_{1} i, j=\frac{\exp \left(-\alpha l_{i}\right)}{\sum_{\substack{\text { source } \\
\text { illuminated } \\
\text { orders }}} \exp \left(-\alpha l_{k}\right)} \\
C_{2} i, j=\frac{\exp \left(-\alpha l_{i}\right)\left(1-\exp \left(-2 \alpha l_{j}\right)\right)}{\sum_{\substack{\text { source } \\
\text { illuminated } \\
\text { orders }}} \exp \left(-\alpha l_{k}\right)} \\
C_{3} i, j=\exp \left(-2 \alpha l_{i}\right) \delta_{i, j}
\end{gathered}
$$

Indices $i$ and $j$ are made with reference to the row and column numbers of $\mathbf{C}$. For example, for a system with two source-illuminated escaping orders, two nonsource-illuminated escaping orders and two confined orders in configuration (a), the confinement matrix would be:

$\mathbf{C}=\left[\begin{array}{cccccc}1 / 2 & 1 / 2 & 1 / 2 & 1 / 2 & \left(1-\mathrm{e}^{-2 \alpha l_{5}}\right) / 2 & \left(1-\mathrm{e}^{-2 \alpha l_{6}}\right) / 2 \\ 1 / 2 & 1 / 2 & 1 / 2 & 1 / 2 & \left(1-\mathrm{e}^{-2 \alpha l_{5}}\right) / 2 & \left(1-\mathrm{e}^{-2 \alpha l_{6}}\right) / 2 \\ 0 & 0 & 0 & 0 & 0 & 0 \\ 0 & 0 & 0 & 0 & 0 & 0 \\ 0 & 0 & 0 & 0 & \mathrm{e}^{-2 \alpha l_{5}} & 0 \\ 0 & 0 & 0 & 0 & 0 & \mathrm{e}^{-2 \alpha l_{5}}\end{array}\right]$ 
Equation (10) is a system of $2 \mathrm{~N}$ linear equations with $2 \mathrm{~N}$ unknowns that, where a solution exists, is readily soluble. The exact form of CR depends on the device and illumination conditions in question. However, as a result of conservation of energy and time reversal invariance, $\mathbf{R}$ must be doubly stochastic for any lossless grating (all rows and all columns sum to 1 - see Appendix for proof). C is clearly left stochastic (all columns sum to 1 ) and hence the product $\mathbf{C R}$ is also left stochastic. This is an important result and ensures that a solution to (10) always exists and, where all entries of $\mathbf{R}$ are strictly positive, is unique up to a scalar multiple [18].

Once $\mathbf{B}^{\text {inc }}$ has been calculated, $\mathbf{B}^{\text {diff }}$ can then be calculated from (10). Repeating the procedure for all sub-manifolds in the illumination cone yields a full description of the angle dependent brightness within the cell. This allows calculation of the quantities described in the following sections.

\subsection{Calculation of the mean path length of light rays inside the solar cell}

The mean path length of light rays inside the solar cell active layer is a geometrical property of the device structure. For its calculation zero absorption in the active layer is therefore assumed. Following Miñano [7], the mean path length enhancement is calculated from:

$$
\frac{\langle l\rangle}{w}=\frac{\int\left(B^{\text {inc }}\left(u_{x}, u_{y}\right)+B^{\mathrm{diff}}\left(u_{x}, u_{y}\right)\right) \frac{\mathrm{d} u_{x} \mathrm{~d} u_{y}}{u_{z}}}{B_{0} \int_{\Omega_{0}} \mathrm{~d} u_{x} \mathrm{~d} u_{y}}
$$

where the numerator integral is made over all directions in the cell and the denominator integral over the illumination cone $\Omega_{0} . B_{0}$ is the brightness of the illuminating light and $B^{\text {inc }}$ and $B^{\text {diff }}$ are calculated using the preceding steps taking $\alpha=0$.

\subsection{Direct calculation of the absorption}

The fraction of incident power absorbed in the solar cell active layer can be calculated directly from: escaping orders fall within the same section. Taking Figure $2 \mathrm{a}$ as an example, only the bottom half of the illumination cone will produce the diffracted order at the very top of the figure, hence this should form a separate section. Convergence testing should be used to decide how many further subdivisions are required to achieve the desired accuracy.

- For a single ray in each sub-manifold, calculate the scattering matrix as defined in Equation (2) for the resulting system of diffracted orders using an appropriate computational method. There are a variety of methods available to calculate the scattering matrix computationally, based on rigorous solution of Maxwell's equations [16].

- Generate the confinement and redistribution matrix following geometrical considerations (taking $\alpha=0$ to calculate the mean path length or $\alpha \neq 0$ to calculate absorption). - Solve (10) and apply (5) to give the brightnesses of incident and diffracted orders. Repeating for each subsection gives a full description of the angle dependent brightness inside the solar cell.

- Apply (16) or (17) to calculate the quantity of interest.

\section{UPPER LIMITS OF LIGHT TRAPPING IN SOLAR CELLS USING DIFFRACTION GRATINGS AND ANGULARLY SELECTIVE RUGATE FILTERS}

\subsection{Light trapping in the weak absorption limit}

If $l$ is the length a ray path travels through the solar cell active layer, the weak absorption limit is defined as the regime wherein all illuminated ray paths satisfy $\alpha l<<1$. Within this regime, absorption in the solar cell relative to the incident power can be approximated to [7]:

$$
A=\alpha\langle l\rangle
$$

where $\langle l\rangle$ is the mean path length of light rays within the active layer. Within the weak absorption limit, a discussion of mean path lengths is therefore sufficient for comparison

$A=\frac{\int\left(\left(\exp \left(\alpha w / u_{z}\right)-1\right) B^{\mathrm{inc}}\left(u_{x}, u_{y}\right)+\left(1-\exp \left(-\alpha w / u_{z}\right)\right) B^{\mathrm{diff}} \overline{\left.\left(u_{x}, u_{y}\right)\right) \mathrm{d} u_{x} \mathrm{~d} u_{y}}\right.}{B_{0} \int_{\Omega_{0}} \mathrm{~d} u_{x} \mathrm{~d} u_{y}}$

\subsection{Procedure for application of the formalism to computational calculations}

The procedure for computational calculation of the absorption and optical mean path length in a solar cell equipped with any proposed grating structure is summarised as follows:

. Divide the illumination cone into sub-manifolds. Firstly, divisions must be made so that all incidence directions which produce the same confined orders, sourceilluminated escaping orders and non-source-illuminated of different light trapping techniques. This is a geometric property; hence we consider the solar cell to be transparent throughout this section.

\subsubsection{Thermodynamic and Lambertian limits.}

The brightness theorem [17] states that the brightness $B_{\text {int }}$ of a given internal ray path is limited to:

$$
B_{\text {int }} \leq n^{2} B_{0}
$$


Miñano [7] states that if all ray paths within the cell are illuminated with the maximum brightness then the mean path length of light rays in a cell illuminated by a light cone of half angle $\theta_{0}$ is:

$$
\langle l\rangle_{\max }=\frac{4 n^{2} w}{\sin ^{2} \theta_{0}}
$$

which represents the thermodynamic upper limit. It should be noted that this result assumes nothing of the light trapping mechanism; it therefore serves as an upper limit for any light trapping scheme. The upper limit for any specific scheme (diffraction grating, Rugate filter, etc.) is already restricted to being no greater than (20), though it may be lower.

In the Lambertian scheme, Yablonovitch and Cody [4] show by detailed balancing of the incident and escaping power flux that the internal brightness is given by:

$$
B_{\text {int }}=n^{2} B_{0} \sin ^{2} \theta_{0}
$$

yielding the mean path length for ideal Lambertian light trapping to be:

$$
\langle\rangle_{\text {Lambertian }}=4 n^{2} w
$$

The factor of $\sin ^{2} \theta_{0}$ by which (21) and (22) are lower than (19) and (20) is the ratio of the étendues of the incident and escaping light. In the absence of angular selectivity, isotropic internal illumination implies isotropic escaping illumination and where incident illumination is not isotropic the escaping and hence internal brightness must be less than the incident brightness to compensate. It should therefore be clear that any light trapping scheme which attempts to supersede the Lambertian scheme must seek to satisfy the following conditions:

Condition 1: maximise the number of ray paths within the cell that are illuminated.

Condition 2: minimize the escaping étendue and hence maximise the brightness of illuminated paths inside the cell (the étendue cannot be decreased by an optical system without light loss; hence the escaping étendue cannot be lower than the incident étendue).

\subsubsection{The upper limit for the Rugate filter.}

A Rugate filter is an angularly-selective photonic crystal which only allows light incident at certain angles to pass through in either direction. It may be applied to light trapping by placing it on the front surface of the solar cell and placing a Lambertian scatterer on the rear [13]. The purpose of the filter is to increase the proportion of internal rays which are internally reflected whilst maintaining transmission of the incident light into the cell. The ideal Rugate filter would admit total transmittance of all light within the incidence cone and total reflectance for all other directions. The escaping étendue would then match exactly the incident étendue, and the vacuum brightness of the escaping light equal the vacuum brightness of the source. The brightness of the escaping light inside the cell is greater than that in a vacuum by a factor of $n^{2}[4]$ and the internal illumination is isotropic by virtue of the Lambertian scatterer. It clearly follows that all internal ray paths would be illuminated with the maximum possible brightness in Equation (19). The upper limit of the mean path length for a Rugate filter therefore coincides with the overall thermodynamic limit in Equation (20).

\subsubsection{The upper limit for a diffraction grating.}

3.1.3.1. Intemal brightness for large $\lambda / \Lambda$. When $\lambda / \Lambda$ is sufficiently large that there are no escaping orders other than the zero-order for all rays in the incidence cone (as illustrated in Figure $2 \mathrm{a}$ and $\mathrm{b}$ ), the middle row of $\mathbf{C}$ in Equation (11) vanishes and $\mathbf{C}$ is doubly stochastic (remembering that $\mathbf{C}_{2}=\mathbf{0}$ and $\mathbf{C}_{3}=\mathbf{I}$ for a transparent cell). For a lossless grating, the product $\mathbf{C R}$ is therefore doubly stochastic and the solution to $(10)$ is that all internal orders have the maximum thermodynamically allowed brightness of $n^{2} B_{0}$. Using a diffraction grating in this $\lambda / \Lambda$ range ensures that the escaping étendue is no greater than the incident étendue; hence it is guaranteed that Condition 2 in Section 3.1.1 is satisfied for any grating profile. This result holds if all elements of $\mathbf{C R}$ are strictly positive, which automatically excludes trivial situations such as a perfect reflector on the front surface, or a grating with no surface modulation (a flat surface).

3.1.3.2. The mean path length for an ideal grating. It should be clear from Figure 2 that it is impossible to illuminate all internal directions without allowing non-zero diffracted orders to escape. To satisfy Condition $l$ it is therefore necessary to work in the lower $\lambda / \Lambda$ range where maximum brightness is not guaranteed. To seek an upper limit for light trapping we must therefore find the maximum brightness which can be achieved in this regime without violating conservation of energy or time reversal invariance. Both constraints combined require that $\mathbf{R}$ be doubly stochastic.

Consider the decomposition of $\mathbf{R}$ into the following block matrix

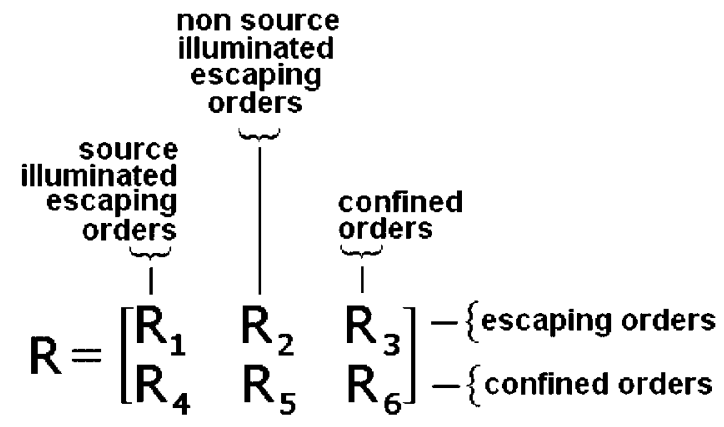


We wish to minimise $\mathbf{R}_{3}$ : the coupling from confined to escaping orders, whilst maximising $\mathbf{R}_{\mathbf{4}}$ : the coupling from source illuminated to confined orders. Double stochasticity of $\mathbf{R}$ requires that the sum of all elements in $\mathbf{R}_{3}$ be at least the sum of all elements in $\mathbf{R}_{4}$ because:

$$
\begin{aligned}
\sum_{\text {columns }}\left[\begin{array}{l}
\mathbf{R}_{3} \\
\mathbf{R}_{6}
\end{array}\right] & =N_{\text {confined }}=\sum_{\text {rows }}\left[\begin{array}{lll}
\mathbf{R}_{4} & \mathbf{R}_{5} & \mathbf{R}_{6}
\end{array}\right] \\
& \Rightarrow \sum_{\text {elements }} \mathbf{R}_{3} \geq \sum_{\text {elements }} \mathbf{R}_{4}
\end{aligned}
$$

Physically this means that the degree of coupling out of the confined orders must be greater than or equal to the degree of coupling into confined orders, there being more orders escaping the cell then illuminated orders coupling into it. Confinement is maximised when these sums are equal which is achieved if $R_{5}=0$ : a zero matrix. The grating then acts as an angle selector; restricting coupling of un-illuminated incident directions into the cell. Under this ideal grating condition we have:

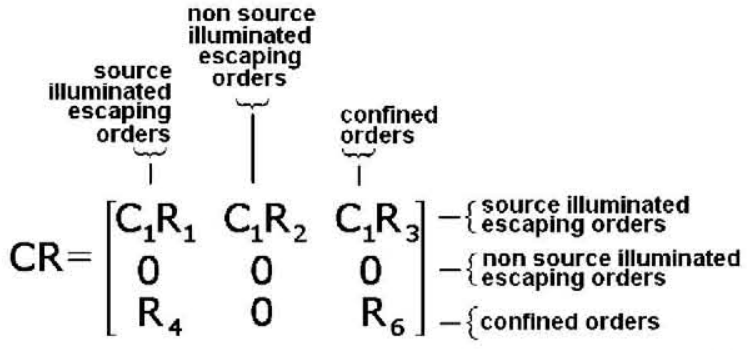

and the solution to (10) is that the brightness in all confined orders is again $n^{2} B_{0}$; the brightness limit for confined orders coincides with the thermodynamic brightness theorem limit. It must be emphasized that no grating is proposed here which would satisfy this condition. It is merely stated that a grating with such a property would offer maximum light trapping without violating the thermodynamic or reciprocal constraints to which diffraction gratings are subject. It is therefore appropriate to refer to such a grating as an ideal grating in a discussion of limits.

In configuration (a) in Figure 1, all orders internal to the cell are confined orders. The mean path length enhancement for an ideal grating placed on the front face is therefore calculated from (16) by considering that the upward and downward travelling brightnesses are $n^{2} B_{0}$ within the diffracted cones and zero everywhere else. The result is dependent on two parameters; firstly, on the number and orientation of cones within the solar cell, which are directly related to the quantities $\left|\overrightarrow{\mathbf{b}}_{1}\right| \lambda_{0} / 2 \pi$ and $\left|\overrightarrow{\mathbf{b}}_{2}\right| \lambda_{0} / 2 \pi$ by the Fraunhofer Equation (1); and secondly, on the half angle illumination cone, which is directly related to the concentration factor $C$ of the illuminating light by $\sin \theta_{0}=\sqrt{C} \sin \theta_{\text {acc }}$ where $\theta_{\text {acc }}$ is the acceptance angle at the concentrator aperture [19] ( $C=1 \times$ corresponds to a flat panel system). We make the simplifying assumption that, for bi-periodic gratings, both periods are equal so that $\left|\overrightarrow{\mathbf{b}}_{1}\right|=\left|\overrightarrow{\mathbf{b}}_{2}\right|=|\overrightarrow{\mathbf{b}}|$.

In Figure 3 mean path length enhancements $\langle l\rangle / w$ for gratings satisfying the ideal grating condition $\left(\mathbf{R}_{5}=0\right)$ placed on the front face are plotted as a function of $|\overrightarrow{\mathbf{b}}| \lambda_{0} / 2 \pi$, where $|\overrightarrow{\mathbf{b}}| \lambda_{0} / 2 \pi=\lambda / \Lambda$ for line and square lattice gratings and $|\overrightarrow{\mathbf{b}}| \lambda_{0} / 2 \pi=(2 / \sqrt{3}) \lambda / \Lambda$ for a triangular lattice grating. Figure $3 a$ shows results for an illumination cone of half angle $\theta_{0}=1^{\circ}$ and Figure $3 \mathrm{~b}$ for $\theta_{0}=33.5^{\circ}$, these correspond to an acceptance angle of $1^{\circ}$ and concentration factors of (a) $1 \times$ and (b) $1000 \times$, respectively. The cell refractive index is taken to be $n=3.33$ corresponding to GaAs. Red, green and blue curves show results for ideal triangular lattice, square lattice and line gratings, respectively, all placed on the cell's front surface. The lattice geometries are pictured in (b), and the internal diffracted cones pictured at certain points on
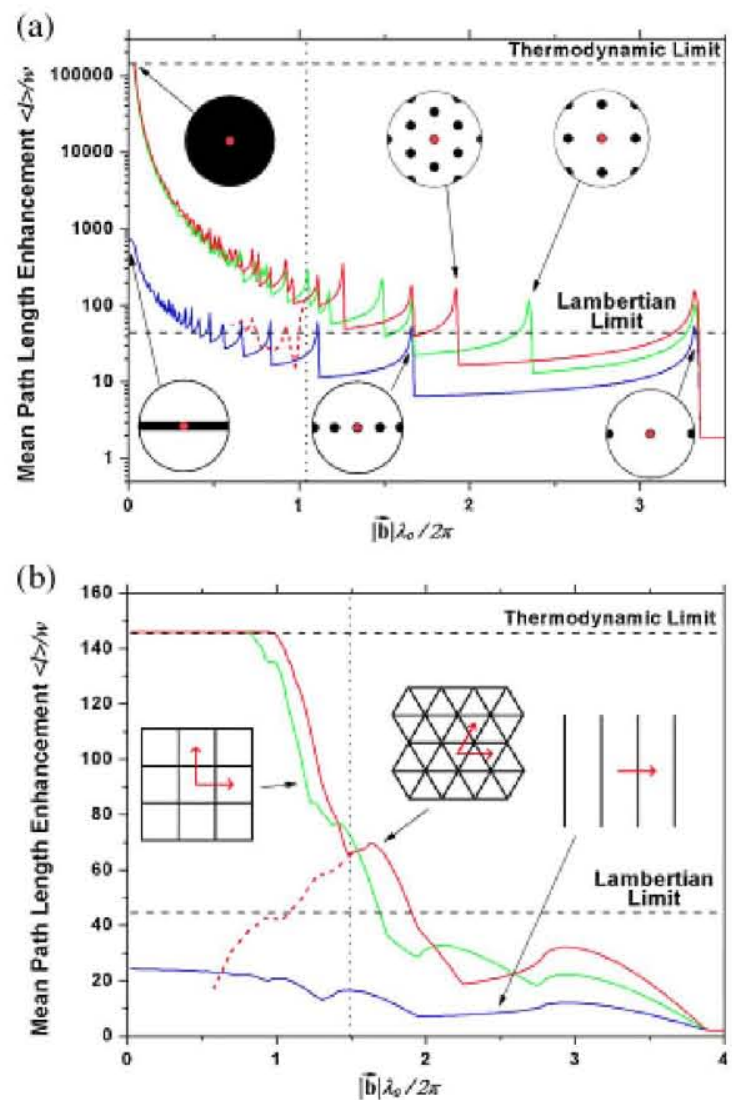

Figure 3. Mean path length enhancement as a function of wavelength to grating period ratio for (a) $1 \times$ and (b) $1000 \times$ concentration and an acceptance angle of $1^{\circ} . n=3.33$ (GaAs) The red, pink and blue curves show path lengths for idea triangular lattice, square lattice and line gratings, respectively The dashed red curves show results from simulation of a grating geometry consisting of a triangular lattice of cylindrical wells. 
the curves in (a). Local peaks occur at points where highly oblique orders are introduced. The thermodynamic limit is achievable by the bi-periodic triangular and square lattice gratings at low $\lambda / \Lambda$ where the diffracted cones fill the cell. This will also hold for $\left|\overrightarrow{\mathbf{b}}_{1}\right| \neq\left|\overrightarrow{\mathbf{b}}_{2}\right|$ since diffracted cones can always be made to fill the cell completely.

The uni-periodic line grating only diffracts light along a single axis and is incapable of filling all directions within the cell with light (see lower left inset in (a)). The upper limit for a line grating is therefore considerably lower than the thermodynamic limit. In fact, at $1000 \times$ the uniperiodic line grating limit is lower than the Lambertian limit.

The range to the right of the vertical dotted line in both figures corresponds to that in which only the zero order escapes and maximum brightness is guaranteed for a transparent cell, as described at the beginning of Section 3.1.3. To the right of the line, the plotted mean path length would be achieved by any grating profile. To the left, it would only be achieved by a grating satisfying $R_{5}=0$ in Equation (23) as previously discussed.

To clarify this, $\langle l\rangle / w$ has been calculated numerically for a simple grating geometry consisting of a triangular lattice of cylindrical wells with well depth $d=0.3 \Lambda$ and well radius $r=0.35 \Lambda$, placed on the solar cell front surface. The scattering matrix elements were calculated using commercial software package $\mathrm{Gd}-\mathrm{Calc}^{\left({ }^{(}\right)}$: an electromagnetic simulation program based on rigorous coupled-wave (RCW) theory, and the formalism of Section 2 was applied. The results are plotted as dashed red curves in Figure $3 \mathrm{a}$ and $\mathrm{b}$. No results are shown for $|\overrightarrow{\mathbf{b}}| \lambda_{0} / 2 \pi<0.5$ as the high number of propagating orders makes calculations computationally costly in this region. Above the vertical dotted line, $\langle l\rangle / w$ for the simulated grating coincides with the ideal grating as discussed. Below the line, it falls dramatically: multiple escaping orders now exist and the simulated grating is not restricting coupling between these and confined orders, causing the internal brightness to decrease. It should be noted that, although the simulated grating fails to approach the thermodynamic limit, a significant improvement is made on the Lambertian limit over a wide wavelength range for both $1 \times$ and $1000 \times$.

These observations have important implications for the practical application of gratings to solar cells. Approaching the ideal grating condition requires the complicated task of designing a grating such that many scattering matrix elements are simultaneously zero or close to zero over a range of wavelengths. It is reasonable to speculate that the profile such a grating would be complex with many different elements in each unit cell. When designing a simple grating for light trapping, it could therefore be advisable to choose the grating period such that the wavelength range of interest falls into or overlaps the region $1<|\overrightarrow{\mathbf{b}}| \lambda_{0} / 2 \pi<2$. Light trapping in this region has much less potential than for lower $\lambda / \Lambda$, but it can be

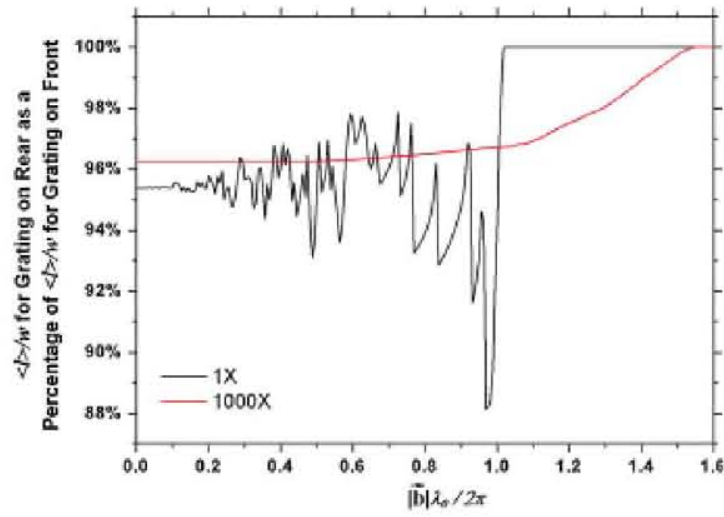

Figure 4. The mean path length for ideal triangular lattice grating placed on the rear surface as a percentage of the mean path length for the same grating placed on the front. Black and red curves show results for $1 \times$ and $1000 \times$ concentrations, respectively.

achieved more easily. More generally, on decreasing $\lambda / \Lambda$, the number of diffracted orders and hence the complexity of the system increases. This makes design more difficult. There is therefore a trade-off between overall potential and achievability in practice.

Finally, when $\lambda / \Lambda$ is very low, diffracted orders become so dense they form a continuum and the geometric optics regime is entered. We argue that as long as the grating profile is sufficiently complex to diffract some light into all directions, it is still correct to state that the grating limit is no lower than the thermodynamic limit.

3.1.3.1. The upper limit for a grating placed on the rear face. Figure 3 shows the upper limit for a grating placed on the front face of the solar cell. If the grating is placed on the rear of the cell, all downward directions within the escape cone but outside the illumination cone are un-illuminated (this is the grey area in Figure 2d). Also the requirement that the ideal grating restrict escaping étendue implies some un-illuminated upward directions within the escape cone. The thermodynamic limit is therefore unachievable by a grating placed on the rear under all conditions other than isotropic illumination of the front surface (ideal concentration). The mean path length enhancements $\langle l\rangle / w$ for a triangular lattice grating satisfying the ideal grating condition $\left(R_{5}=0\right)$ when placed on the rear face is shown in Figure 4 as a percentage of the same grating placed on the front, for $1 \times$ and $1000 \times$ concentrations. The upper limit (achieved at low $\lambda / \Lambda$ ) for a grating placed on the rear face is $4.6 \%$ and $3.7 \%$ lower than the limit for a grating placed on the front at $1 \times$ and $1000 \times$ concentrations, respectively.

\subsection{Light trapping outside of the weak absorption limit}

Outside the weak absorption limit, the mean path length alone is not sufficient to describe total absorption. Using 
the properties of the exponential function, it is shown in Ref. [7] that for a given mean path length, cells with a narrow distribution of path lengths will absorb more than cells with a wide distribution. Hence we have a third condition to achieve maximum absorption outside the weak absorption limit:

Condition 3: minimize the variance of the distribution of path lengths within the cell.

\subsubsection{The thermodynamic limit.}

The maximum possible absorption is achieved when the internal rays have the maximum mean path length from (20) and when all rays travel the same length [7]. The thermodynamic limit of total absorption relative to the power incident on the cell is then

$$
A_{\max }=1-\exp \left(-\alpha\langle l\rangle_{\max }\right)
$$

\subsubsection{The upper limit for a diffraction grating.}

For a single incident ray, condition 3 is fulfilled by any grating whose redistribution matrix $\mathrm{R}$ consists of zeroes and ones (a single one in each row and column and zeroes elsewhere; this does not violate double stochasticity of $\mathrm{R}$ - also, note that this implicitly implies an asymmetric grating). A single path is then followed which, if $R_{5}=0$ as before, will traverse all confined orders. If a single system of diffracted orders has multiple source illuminated orders, then these incident rays must divide the diffracted paths between them. Condition 3 is best fulfilled when the set of diffracted paths is most evenly partitioned between the source illuminated orders. For a given system this partition cannot necessarily be perfectly even. What's more, each ray in the illumination cone corresponds to a different system with different associated path lengths. This introduces the possibility for path length variance. It is not, therefore, trivial that the thermodynamic limit is achievable.

The fraction of incident power absorbed by a cell equipped with an ideal grating has been calculated as a function of $\lambda / \Lambda$ using an algorithm. The incidence cone is divided into a finite number of rays and the absorption calculated for each by summing the path lengths of the corresponding diffracted orders and applying the Lambert-Beer law. Where multiple rays pertain to a single system, the diffracted paths are partitioned between them using a 'greedy' algorithm. This chooses the largest path in the system and puts it in the emptiest partition then does the same with the next largest and so on. The greedy algorithm does not guarantee an optimum partition but this can only serve to underestimate the result.

The calculated absorptions are shown in Figure 5 for (a) $1 \times$ and (b) $1000 \times$ concentration. The acceptance angle and refractive index are $1^{\circ}$ and $n=3.33$, respectively, as before. Results for absorbances of $\alpha w=1 \times 10^{-4}, \alpha w=$ $1 \times 10^{-3}, \alpha w=0.01$ and $\alpha w=0.1$ are displayed in black,
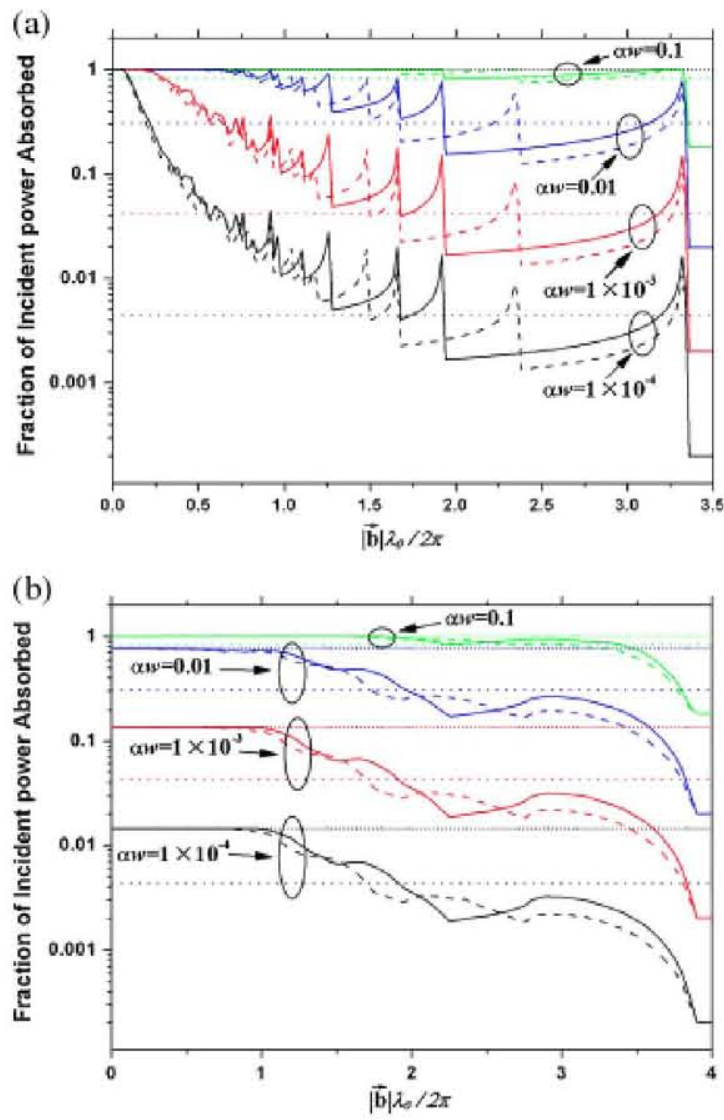

Figure 5. Absorption as a function of wavelength to grating period ratio for (a) $1 \times$ and (b) $1000 \times$ concentration and an acceptance angle of $1^{\circ} . n=3.33$ (GaAs). Calculated absorptions in cells with absorbances of $\alpha W=1 \times 10^{-4}, \alpha W=1 \times 10^{-3}, \alpha W=0.01$ and $\alpha W=0.1$ are displayed in black, red, blue and green, respectively. Solid curves and dashed curves show absorptions achieved using ideal triangular lattice and square lattice gratings, respectively. In each case the grating is placed on the cell's front surface. Closely spaced and widely spaced dotted lines show thermodynamic and Lambertian limits, respectively.

red, blue and green, respectively. In each case the absorption for a triangular lattice grating (solid line), a square lattice grating (dashed line), the respective thermodynamic limit (closely spaced dotted line) and Lambertian limit (widely spaced dotted line) is plotted. For $1 \times$ concentration the thermodynamic absorption limit is unity for all absorbances.

In all studied cases the absorption reaches the corresponding thermodynamic limit within a relative margin of $0.25 \%$ at low $\lambda / \Lambda$. This can be explained as follows: As diffracted orders become dense within the cell, the summed path lengths for each system of diffracted orders tend to a common value. Additionally, the number of source illuminated orders in each system increases exponentially while the difference between the number of source illuminated orders in different systems remains of the same order of magnitude. Hence each system has a 


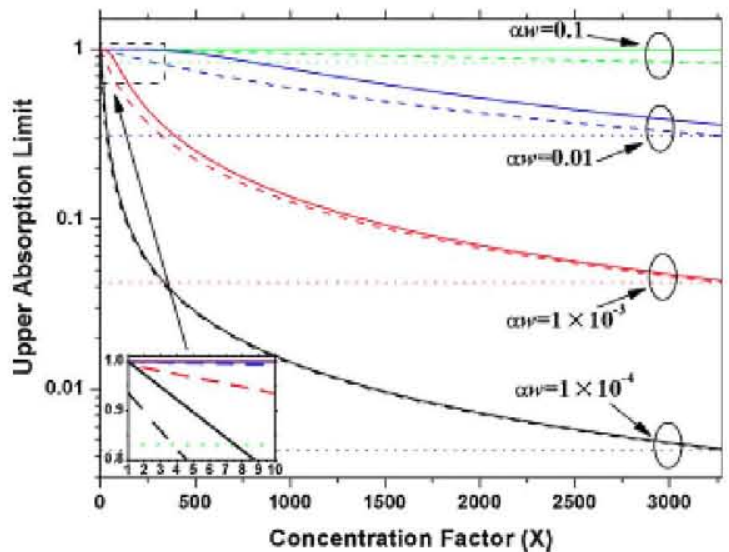

Figure 6. The upper absorption limits for cells employing a diffraction grating placed on the front face (solid lines) and a Rugate filter (dashed lines) as a function of the concentration factor of the illuminating light. Limits for cells with absorbances of $\alpha w=1 \times 10^{-4}, \alpha w=1 \times 10^{-3}, \alpha w=0.01$ and $\alpha w=0.1$ are displayed in black, red, blue and green, respectively. In each case the grating is placed on the cell's front surface. Dotted lines represent the Lambertian limit for each absorbance.

relatively similar number of source illuminated orders; and as there are many diffracted orders, their lengths may be evenly partitioned. The total variance in path lengths can then be made to be arbitrarily small: the upper absorption limit for a diffraction grating is equal to the thermodynamic limit. The solid lines in Figure 6 represent this limit as a function of concentration factor for the aforementioned absorbances. The concentration factor is directly related to the half angle of the illumination cone as mentioned earlier. An acceptance angle of $1^{\circ}$ is again assumed.

\subsubsection{The Lambertian limit and the upper limit for the Rugate filter.}

An analytical method of calculating the absorption for a cell equipped with a Lambertian scatterer has been presented by Green [20]. This can be extended to include the effect of an ideal Rugate filter by simply setting the escaping étendue to be equal to the incident étendue in Green's formula. The upper absorption limits for Lambertian trapping and for the Rugate filter are plotted in Figure 6 as dotted and dashed lines, respectively. For higher absorbances the upper Rugate filter limit falls increasingly short of the diffraction grating/thermodynamic limit. This is a result of the inevitable variance in path length distribution caused by the random scattering employed in this scheme.

\subsection{Discussion}

Our findings are summarised in Figure 6. Both the Rugate filter and diffraction grating limits demonstrate a significant improvement on the Lambertian limit. This becomes more pronounced at lower concentrations. It should be noted that concentration factors currently employed in practical systems rarely exceed $1000 \times$.

It has been demonstrated that, for both the Rugate filter and front face diffraction grating schemes, the upper limit of the geometrical mean path length is equal to its thermodynamic limit. Hence, for low absorbances, where mean path length is the dominant factor, the limits of both schemes are similar.

The thermodynamic absorption limit is only achievable by the diffraction grating; the Rugate filter absorption limit is generally lower. This difference is significant for all but extremely weakly absorbing cells (even for $\alpha w=1 \times 10^{-4}$ at $1 \times$ concentration the Rugate limit is still $6 \%$ lower than the diffraction grating limit - see inset Figure 6). The essential difference is that although both components are capable of acting as an angle selector at the front surface, only the diffraction grating has the ability to control the paths of light rays inside the cell substrate.

Finally, although it has been demonstrated that the diffraction grating has the higher limit of the two schemes, this does not necessarily imply that it will achieve better light trapping in practice. The constraints placed to the grating to achieve maximum absorption are extremely demanding. It is not known what profile a grating which approximated to the ideal case would have or if indeed such a profile exists. Of course optimising a Rugate filter to restrict redistribution to the incidence cone perfectly is no minor task either, although some good results have been achieved [21]. In stating the upper limits explicitly, we hope to have reiterated the motivation for research in both fields.

\section{CONCLUSIONS}

A mathematical formalism has been derived to calculate the mean path length enhancement and absorption in a solar cell equipped with a given diffraction grating of given profile and known scattering matrices. To the authors knowledge no such formalism has existed to date. Applying this formalism, the upper absorption limits of light trapping using a diffraction grating has been calculated. The upper limit using a Rugate filter has also been calculated analytically. The derived formalism and limits are valid when the solar cell thickness is greater than the coherence length of the illuminating solar spectrum. Both limits surpass that of conventional Lambertian light trapping. The increase is considerable within the range of practically implemented concentration factors. The upper absorption limit using a bi-periodic diffraction grating placed on the front cell surface is equal to the thermodynamic limit for all concentration factors and cell absorbances. The upper limit using the Rugate filter is generally lower but tends to the thermodynamic limit at low absorbances. The upper limits for a bi-periodic grating placed on the rear of the cell and for a bi-periodic line grating placed on either surface are marginally and 
considerably lower than the thermodynamic limit, respectively. Diffraction gratings have greater light trapping potential in the higher grating period range where many propagating diffracted orders are generated within the solar cell, the limit only being achievable when diffracted cones fill the cell completely.

\section{ACKNOWLEDGEMENTS}

This work was supported by the European Commission project IBPOWER (Contract 211640), the NUMANCIA (S2009/ENE/1477) project funded by the Comunidad de Madrid, and the Nanogeffes project, which is part of the Spanish National Program. Alexander Mellor gratefully acknowledges the Comunidad de Madrid for financial support through the scholarship Personal Investigador de Apoyo.

\section{REFERENCES}

1. Luque A, Martí A. The intermediate band solar cell: Progress toward the realization of an attractive concept. Advanced Materials 22(2): 160-174.

2. Marti A, Lopez N, Antolin E, Canovas E, Stanley C, Farmer C, Cuadra L, Luque A. Novel semiconductor solar cell structures: The quantum dot intermediate band solar cell. Thin Solid Films 2006; 511: 638-644.

3. Deckman HW, Wronski CR, Witzke H, Yablonovitch E. Optically enhanced amorphous-silicon solar-cells. Applied Physics Letters 1983; 42(11): 968-970.

4. Yablonovitch E, Cody GD. Intensity enhancement in textured optical sheets for solar-cells. IEEE Transactions on Electron Devices 1982; 29(2): 300-305.

5. Fornies E, Zaldo C, Albella JM. Control of random texture of monocrystalline silicon cells by angleresolved optical reflectance. Solar Energy Materials and Solar Cells 2005; 87(1-4): 583-593.

6. Campbell P, Green MA. Light trapping properties of pyramidally textured surfaces. Journal of Applied Physics 1987; 62(1): 243-249.

7. Miñano JC. In Physical Limitations to Photovoltaic Energy Conversion, Luque A, Araujo G (eds). Adam Hilger: Bristol, 1990; Ch. 3, pp. 50-55.

8. Zhao JH, Wang AH, Campbell P, Green MA. A $19.8 \%$ efficient honeycomb multicrystalline silicon solar cell with improved light trapping. IEEE Transactions on Electron Devices 1999; 46(10): 1978-1983.

9. Zhao JH, Wang AH, Green MA. $24.5 \%$ efficiency silicon PERT cells on MCZ substrates and $24.7 \%$ efficiency PERL cells on FZ substrates. Progress in Photovoltaics 1999; 7(6): 471-474.

10. Abouelsaood AA, El-Naggar SA, Ghannam MY. Shape and size dependence of the anti-reflective and light-trapping action of periodic grooves. Progress in Photovoltaics 2002; 10(8): 513-526.

11. Sai H, Kanamori Y, Arafune K, Ohshita Y, Yamaguchi M. Light trapping effect of submicron surface textures in crystalline Si solar cells. Progress in Photovoltaics $2007 ; 15(5): 415-423$.

12. Llopis F, Tobias I. Influence of texture feature size on the optical performance of silicon solar cells. Progress in Photovoltaics 2005; 13(1): 27-36.

13. Fahr S, Ulbrich C, Kirchartz T, Rau U, Rockstuhl C, Lederer F. Rugate filter for light-trapping in solar cells. Optics Express 2008; 16(13): 9332-9343.

14. Tobias I, Luque A, Marti A. Light intensity enhancement by diffracting structures in solar cells. Journal of Applied Physics 2008; 104(3): 034502.

15. Nevière M. In Electromagnetic Theory of Gratings (Topics in Applied Physics), Petit R (ed.). Springer: Berlin, 1980; Ch. 5, pp. 145-147.

16. Neviere M, Popov E. Light Propagation in Periodic Media: Differential Theory and Design (Optical Engineering). Marcel Dekker: New York, 2002.

17. Born M, Wolf E. Principles of Optics: Electromagnetic Theory of Propagation, Interference and Diffraction of Light, (7th Edition). Cambridge University Press: Cambridge, 1999.

18. Berman A, Plemmons R. Nonnegative Matrices in the Mathematical Sciences (Classics in Applied Mathematics). Society for Industrial Mathematics: Philadelphia, 1994.

19. Swanson RM. In Handbook of Photovoltaic Science and Engineering, Luque A, Hegedus S (eds). Wiley: Chichester, 2003; Ch. 11, pp. 474-475.

20. Green MA. Lambertian light trapping in textured solar cells and light-emitting diodes: Analytical solutions. Progress in Photovoltaics 2002; 10(4): 235-241.

21. Peters M, Goldschmidt JC, Kirchartz T, Blasi B. The photonic light trap-improved light trapping in solar cells by angularly selective filters. Solar Energy Materials and Solar Cells 2009; 93(10): 1721-1727.

\section{APPENDIX: PROOF THAT THE REDISTRIBUTION MATRIX $R$ IS DOUBLY STOCHASTIC}

The redistribution matrix $\mathrm{R}$ is defined by (10) in the main body of the text as

$$
R_{i, j}=\frac{n_{i} u_{z i}}{n_{j} u_{z j}}\left|S_{i, j}\right|^{2}
$$

We wish to show that

$$
\sum_{i} R_{i, j}=1 \forall j
$$

and

$$
\sum_{j} R_{i, j}=1 \forall i
$$

Proof:

The total power incident on the grating is:

$$
P_{\text {inc }}=\sum_{j} n_{j} u_{z j}\left|E_{j}^{\mathrm{inc}}\right|^{2}
$$


If $E_{i n c}$ is a column vector with elements $E_{j}^{\text {inc }}$ (not to be confused with the directional electric field vector $\overrightarrow{\mathbf{E}}$ ) then:

$$
\mathbf{P}_{i n c}=\mathbf{E}_{i n c}^{\dagger} \mathbf{U \mathbf { E } _ { i n c }}
$$

where $\nmid$ denotes the adjoint matrix. If $\mathbf{E}_{\text {diff }}$ is similarly defined then the total power leaving the grating is:

$$
\mathbf{P}_{\text {diff }}=\mathbf{E}_{\text {diff }}^{\dagger} \mathbf{U} \mathbf{E}_{\text {diff }}
$$

For a lossless grating $\mathrm{P}_{\text {diff }}=\mathrm{P}_{\text {inc }}$ so that:

$$
\begin{aligned}
\mathbf{P}_{\text {diff }} & =\mathbf{E}_{d i f f}^{\dagger} \mathbf{U} \mathbf{E}_{d i f f}=\left(\mathbf{S} \mathbf{E}_{i n c}\right)^{\dagger} \mathbf{U}\left(\mathbf{S} \mathbf{E}_{i n c}\right) \\
& =\mathbf{E}_{i n c}^{\dagger}\left(\mathbf{S}^{\dagger} \mathbf{U S}\right) \mathbf{E}_{i n c}=\mathbf{E}_{i n c}^{\dagger}(\mathbf{U}) \mathbf{E}_{i n c}=\mathbf{P}_{i n c}
\end{aligned}
$$

hence:

$$
\mathbf{S}^{\dagger} \mathbf{U S}=\mathbf{U}
$$

or

$$
\begin{gathered}
\sum_{i}\left(\sum_{k} S_{i, k}^{\dagger} U_{k, i}\right) S_{i, j}=U_{i, j} \\
U_{i, j}=\delta_{i, j} n_{i} u_{z i} \text { and } S_{i, j}^{\dagger}=S_{j, i}^{*} \text { so: } \\
\sum_{i} n_{i} u_{z i} S_{i, l}^{*} S_{i, j}=\delta_{l, j} n_{j} u_{z j} \Rightarrow \sum_{i} \frac{n_{i} u_{z i}}{n_{j} u_{z j}}\left|S_{i, j}\right|^{2}=1 \forall j
\end{gathered}
$$

which proves (A2).

Maxwell's equations are invariant under time inversion. If the vector field in the ith diffracted polarized order is:

$$
\overrightarrow{\mathbf{E}}_{\mathbf{i}}(\overrightarrow{\mathbf{r}}, t)=\operatorname{Re}\left[E_{i} \exp \left(i\left(\overrightarrow{\mathbf{k}}_{i} \cdot \overrightarrow{\mathbf{r}}-\omega t\right)\right)\right] \overrightarrow{\mathbf{v}}_{i}
$$

where $\overrightarrow{\mathbf{v}}_{i}$ is the unit polarization vector and non-italic $i$ is the imaginary unit, then the time reversed field is:

$$
\begin{aligned}
\vec{E}_{i}^{\prime}\left(\overrightarrow{\mathbf{r}}, t^{\prime}\right) & =\vec{E}_{i}\left(\overrightarrow{\mathbf{r}},-t^{\prime}\right) \\
& =\operatorname{Re}\left[E_{i} \exp \left(i\left(\overrightarrow{\mathbf{k}}_{i} \cdot \overrightarrow{\mathbf{r}}+\omega t^{\prime}\right)\right)\right] \overrightarrow{\mathbf{v}}_{i} \\
& =\operatorname{Re}\left[E_{i}^{*} \exp \left(i\left(\overrightarrow{\mathbf{k}}_{i}^{\prime} \cdot \overrightarrow{\mathbf{r}}-\omega t^{\prime}\right)\right)\right] \overrightarrow{\mathbf{v}}_{i}
\end{aligned}
$$

where $\overrightarrow{\mathbf{k}}_{i}^{\prime}=-\overrightarrow{\mathbf{k}}_{i}$. The diffracted orders in the original system are incident orders in the new system and vice versa. Their amplitudes are the complex conjugates of those in the original system. $\overrightarrow{\mathbf{E}}_{\text {inc }}^{\prime}=\overrightarrow{\mathbf{E}}_{\text {diff }}^{*}$ and $\overrightarrow{\mathbf{E}}_{\text {diff }}^{\prime}=\overrightarrow{\mathbf{E}}_{\text {inc }}^{*}$. If $S^{\prime}$ is the scattering matrix of the time reversed system then:

$$
\overrightarrow{\mathbf{E}}_{i n c}^{*}=\overrightarrow{\mathbf{E}}_{\text {diff }}^{\prime}=\mathbf{S}^{\prime} \overrightarrow{\mathbf{E}}_{i n c}^{\prime}=\mathbf{S}^{\prime} \overrightarrow{\mathbf{E}}_{\text {diff }}^{*} \Rightarrow \mathbf{S}^{\prime}=\left(\mathbf{S}^{-1}\right)^{*}
$$

Equation (A8) applies to $S$ ' as it must conserve power (U remains unchanged under time reversal).

$$
\mathbf{S}^{\dagger} \mathbf{U} \mathbf{S}^{\prime}=\mathbf{U}
$$

substituting (A13) into (A14) and performing some operations we have:

$$
\mathbf{S U}^{-1} \mathbf{S}^{\dagger}=\mathbf{U}^{-1}
$$

Following similar steps to (A9) and (A10) gives:

$$
\sum_{j} \frac{n_{i} u_{z i}}{n_{j} u_{z j}}\left|S_{i, j}\right|^{2}=1 \forall i
$$

proving (A3). Hence R is doubly stochastic. 